\title{
ANTIBIOTIC SUSCEPTIBILITY OF SULFAMETHOXAZOLE-TRIMETHOPRIM RESISTANT STENOTROPHOMONAS MALTOPHILIA STRAINS ISOLATED AT A TERTIARY CARE CENTRE IN HUNGARY
}

\author{
EMESE JUHÁSZ*, JÚLIA PONGRÁCZ, \\ MIKLÓS IVÁN and KATALIN KRISTÓF \\ Diagnostic Laboratory of Clinical Microbiology, Institute of Laboratory Medicine, \\ Semmelweis University, Budapest, Hungary
}

(Received: 28 May 2015; accepted: 9 June 2015)

\begin{abstract}
Sulfamethoxazole-trimethoprim (SXT) is the drug-of-choice in Stenotrophomonas maltophilia caused infections. There has been an increase in resistance to SXT of S. maltophilia over recent years. In this study $30 \mathrm{~S}$. maltophilia clinical isolates resistant to SXT were investigated. Antibiotic susceptibilities for ciprofloxacin, moxifloxacin, levofloxacin, doxycycline, tigecycline, ceftazidime, colistin and chloramphenicol were determined by broth microdilution method. None of the strains were susceptible to ciprofloxacin, tigecycline, ceftazidime or colistin. Only $37 \%$ of the isolates were susceptible to levofloxacin or moxifloxacin. Two isolates resistant to all tested antibiotic agents and two others susceptible only to doxycycline were further investigated: susceptibility for combinations of antibiotics was analyzed by checkerboard technique. According to the fractional inhibitory concentration indices calculated, moxifloxacin plus ceftazidime combination was found to be synergistic in each case. Genetic testing revealed the predominance of sull gene. Our study concluded that the range of effective antibiotic agents is even more limited in infections caused by SXT-resistant $S$. maltophilia. In these cases, in vitro synergistic antibiotic combinations could be potential therapeutic options.
\end{abstract}

Keywords: Stenotrophomonas maltophilia, sulfamethoxazole-trimethoprim resistance, antibiotic resistance, synergism

*Corresponding author; E-mail: juhasz.emese@med.semmelweis-univ.hu 


\section{Introduction}

Stenotrophomonas maltophilia is an emerging, multidrug-resistant, Gramnegative pathogen. After Pseudomonas aeruginosa and Acinetobacter baumannii, it is the third most frequent non-fermenting Gram-negative bacteria causing nosocomial infections [1]. Pneumonia and bacteraemia are the most common clinical presentations of $S$. maltophilia infection, related to high mortality rate (14-69\%) [2]. It causes infections predominantly in hospitalized and immunocompromised patients, but community-acquired infections have also been reported. Differentiation of colonization or infection by $S$. maltophilia can be difficult, especially if bacteria are isolated from respiratory tract samples. Moreover, $S$. maltophilia is often isolated from polymicrobial infections, in which its role is difficult to evaluate. Due to its extended inherent antibiotic resistance (characterized by production of L1-, L2- $\beta$-lactamases, extended spectrum $\beta$-lactamases, multidrug-efflux systems, low permeability of outer membrane, modifing enzymes and target site modifications), therapeutic options are strongly limited. The recommended first-line agent is sulfamethoxazole-trimethoprim (SXT). According to European Committee on Antimicrobial Susceptibility Testing (EUCAST), SXT is the only antibiotic agent currently that can be interpreted in microbiology results, based on established $S$. maltophilia specific clinical breakpoints [3].

Resistance to SXT has been reported mainly due to modified target genes sull (carried by class 1 integrons) and sul2 (carried by insertion sequence common region elements) $[4,5]$. Resistance rates of SXT ranges from 2 to $10 \%$ globally; however studies from Turkey (15\%), Spain (27\%), Taiwan (25\%) and China (30-48\%) reported higher resistance rates [4, 6-8]. In patients suffering from cystic fibrosis (CF), the frequency of SXT-resistant S. maltophilia is higher (24$84 \%$ ) [2, 9-11]. Resistance level of isolates from patients with cancer and those treated in intensive care unit (ICU) might be also higher [4]. In case of SXT resistance - as well as in cases when SXT cannot be used due to hypersensitivity of the patient or adverse effects in high dose therapy - alternative agents have to be taken into consideration. Fluoroquinolones, tetracycline derivates, colistin, ceftazidime and chloramphenicol can have activity against $S$. maltophilia, among few other antibiotic agents [1].

Aims of this study were to test the antibiotic susceptibility of $S$. maltophilia isolates resistant to SXT, to examine the in vitro effect of few antibiotic combinations and to reveal the presence of the most frequent SXT-resistance coding genes. 


\section{Materials and Methods}

Our study included 30 consecutive non-duplicate $S$. maltophilia isolates collected in a five-year period (2010-2014) in the Diagnostic Laboratory of Clinical Microbiology, Institute of Laboratory Medicine, Semmelweis University (Budapest, Hungary). Isolates were identified by conventional methods and MALDI-TOF mass spectrometry (Bruker Daltonics, Bremen) technique. Isolates were cultured from blood $(n=2)$, bronchoalveolar lavage sample $(n=8)$, tracheal aspirate $(n=3)$, sputum $(n=3)$, endotracheal tube $(n=3)$, rectal swab $(n=7)$, urine $(\mathrm{n}=1)$, ear $\operatorname{swab}(\mathrm{n}=1)$ and throat $\operatorname{swab}(\mathrm{n}=2)$. The most of the isolates $(n=23)$ were considered clinically to be colonizer or contaminant bacteria. Only seven isolates were infective as they caused lower respiratory tract infections and bacteraemia. Thirteen strains were isolated from neonates cared in neonatal intensive care unit (ICU), six strains from adult patients treated in ICUs.

The minimal inhibitory concentrations (MICs) of nine antibiotics were determined by broth microdilution method in cation-adjusted Mueller-Hinton broth, according to CLSI testing conditions [12]. The antibiotics tested included $0.5-256 \mathrm{mg} / \mathrm{L}$ SXT (Ratiopharm), 0.5-256 mg/L ciprofloxacin (Fresenius Kabi), 0.064-32 mg/L moxifloxacin (Bayer Pharma), 0.064-32 mg/L levofloxacin (TEVA), $1-512 \mathrm{mg} / \mathrm{L}$ colistin (Sigma-Aldrich), 0.064-32 mg/L doxycycline (Pfizer), 0.064-32 mg/L tigecycline (Wyeth), 1-512 mg/L ceftazidime (Fresenius Kabi) and $0.5-256 \mathrm{mg} / \mathrm{L}$ chloramphenicol (Sigma-Aldrich). While EUCAST has S. maltophilia specific breakpoint only for SXT, the non-species related breakpoints were applied for fluoroquinolones, ceftazidime and tigecycline. For doxycycline - due to absence of non-species related breakpoints - the epidemiological cut-off (ECOFF) value of S. maltophilia was applied. For colistin and chloramphenicol - lacking non-species related breakpoints and approved ECOFF the Pseudomonas sp. specific colistin breakpoint and the S. maltophilia specific Clinical Laboratory Standards Institute (CLSI) chloramphenicol breakpoints were used, respectively. Escherichia coli ATCC 25922, Pseudomonas aeruginosa ATCC 27853 and Staphylococcus aureus ATCC 29213 were used as quality control strains, which correspond with the quality control recommendation of CLSI.

The 20 different antibiotic combinations tested for extremely resistant S. maltophilia strains were the following: a) ceftazidime plus ciprofloxacin, moxifloxacin, levofloxacin, doxycycline, tigecycline, colistin, SXT; b) colistin plus ciprofloxacin, moxifloxacin, levofloxacin, doxycycline, SXT; c) tigecycline plus ciprofloxacin, moxifloxacin, levofloxacin, colistin, SXT; d) SXT plus ciprofloxacin, moxifloxacin, levofloxacin. Antibiotic combinations were analysed by checkerboard technique and in selected cases (when synergy was detected) by E-test 
combination method and disc agar diffusion method, too [13, 14]. Fractional inhibitory concentration indices (FICI) were calculated following the formula described in microbiology procedure guidelines [15]. The summation of FICIs of two antibiotics tested define the effects of antimicrobial agent combinations as antagonistic $(\Sigma$ FICI $>4)$, indifferent $(0.5<\Sigma$ FICI $\leq 4)$ or synergistic $(\Sigma$ FICI $\leq$ $0.5)$. Susceptible breakpoint indices (SBPI) were also used to interpret the results. SBPI was calculated as follows: SBPI = (susceptible breakpoint of antibiotic agent $\mathrm{A}$ / MIC of antibiotic agent $\mathrm{A}$ in combination) + (susceptible breakpoint of antibiotic agent B / MIC of antibiotic agent B in combination). An SBPI of 2 indicates that the $\mathrm{MIC}$ values of the two antibiotics tested in combination are either equivalent to their respective susceptible breakpoints or the MIC of one of the antimicrobials in a combination is less than its susceptible breakpoint. Greater SBPI values indicate more effective combinations [11].

Enterobacterial Repetitive Intergenic Consensus PCR (ERIC-PCR) was used for molecular typing of isolates, as described by Silbert et al. [16]. Isolates from the same ward were tested within the same PCR reaction and in the same gel electrophoreses run. Band patterns generated by ERIC-PCR were visually evaluated in the absence of appropriate software. Isolates that differed by two or more bands were interpreted as unrelated.

The occurrence of two frequent genes coding sulfamethoxazole resistance: sull and sul2, and the presence of class I integron were tested by PCR as described previously [17].

\section{Results}

The MIC values, MIC ranges, breakpoints and interpretations are summarized in Table I. All isolates tested were resistant to SXT, however, MIC values ranged between $8-128 \mathrm{mg} / \mathrm{L}$. None of the isolates was susceptible to ciprofloxacin, tigecycline, ceftazidime or colistin. Only $37 \%$ of them were susceptible to levofloxacin or moxifloxacin. Interpreting isolates with MIC value under ECOFF as susceptible, $50 \%$ of the strains were susceptible to doxycycline. Thirteen $\%$ of the isolates were susceptible to chloramphenicol.

Two isolates resistant to all tested antibiotic agents and two others susceptible only to doxycycline were detected. These four isolates were further investigated: susceptibilities for combinations of antibiotics were tested. The calculated FICIs from the lowest MIC values are shown in Table II. Most of the combinations had indifferent effect. Antagonistic combination was not found. Synergistic effect at least in one isolate was found in combinations of a) ceftazidime plus ciprofloxacin, moxifloxacin, levofloxacin or colistin, b) colistin plus ciprofloxa- 
Table I. Summary of MIC values and interpretations

\begin{tabular}{l|ccc|ccc}
\hline $\begin{array}{l}\text { Antimicrobial agent : } \\
\text { breakpoints (mg/L)* }\end{array}$ & \multicolumn{3}{|c|}{ MIC (mg/L) } & \multicolumn{3}{c}{ \% of isolates } \\
& MIC range & MIC 50 & MIC 90 & Resistant & Intermediate & Susceptible \\
\hline $\begin{array}{l}\text { TMP-SMX : } \\
\text { S } \leq 4, \mathrm{R}>4\end{array}$ & $8-128$ & 32 & 64 & 100 & 0 & 0 \\
$\begin{array}{l}\text { ciprofloxacin : } \\
\text { S } \leq 0.5, \mathrm{R}>1\end{array}$ & $1-32$ & 4 & 16 & 83 & 17 & 0 \\
$\begin{array}{l}\text { levofloxacin : } \\
\mathrm{S} \leq 1, \mathrm{R}>2\end{array}$ & $0.5-16$ & 2 & 8 & 20 & 43 & 37 \\
$\begin{array}{l}\text { moxifloxacin : } \\
\mathrm{S} \leq 0.5, \mathrm{R}>1\end{array}$ & $0.125-8$ & 1 & 4 & 23 & 40 & 37 \\
doxycycline : ** & $0.5-64$ & 4 & 16 & 33 & 17 & 50 \\
$\begin{array}{l}\text { tigecycline : } \\
\mathrm{S} \leq 0.25, \mathrm{R}>0.5\end{array}$ & $0.5-8$ & 2 & 4 & 97 & 3 & 0 \\
$\begin{array}{l}\text { ceftazidime : } \\
\mathrm{S} \leq 4, \mathrm{R}>8\end{array}$ & $16-512$ & 128 & 512 & 100 & 0 & 0 \\
colistin : S $\leq 4, \mathrm{R}>4$ & $8->512$ & 128 & $>512$ & 100 & 0 & 0 \\
$\begin{array}{l}\text { chloramphenicol : } * * * \\
\mathrm{~S} \leq 8, \mathrm{R} \geq 32\end{array}$ & $8-64$ & 16 & 32 & 44 & 43 & 13 \\
\hline
\end{tabular}

*Breakpoints according to EUCAST. SXT breakpoints are specific for S. maltophilia.

Fluoroquinolone, tigecycline and ceftazidime breakpoints are non-species related. Colistin breakpoint is Pseudomonas sp. specific.

**ECOFF of doxycycline is $8 \mathrm{mg} / \mathrm{L}$.

***Breakpoints according to CLSI.

cin, moxifloxacin, levofloxacin or doxycycline, c) tigecycline plus colistin, and d) SXT plus moxifloxacin or levofloxacin. Ceftazidime plus moxifloxacin and ceftazidime plus colistin were the only combinations that showed synergistic effect on each tested isolate. Taking into consideration the peak serum levels during antibiotic treatment (ceftazidime: $60 \mathrm{mg} / \mathrm{L}$, ciprofloxacin: $1.8-4.6 \mathrm{mg} / \mathrm{L}$, levofloxacin: $5.7-8.6 \mathrm{mg} / \mathrm{mL}$, moxifloxacin: $4.5 \mathrm{mg} / \mathrm{L}$, colistin: $5-7.5 \mathrm{mg} / \mathrm{L}$, SXT: 1-2/40-60-9/105 mg/L, tigecycline: $0.63 \mathrm{mg} / \mathrm{L}$, doxycycline: $1.5-2.1 \mathrm{mg} / \mathrm{L}$, chloramphenicol: $11-18 \mathrm{mg} / \mathrm{L}$ ), only the combinations of ceftazidime plus quinolons and colistin plus quinolones can be accepted as synergistic, in certain cases. Furthermore, if EUCAST susceptibility breakpoints are also taken into consideration, just the MIC values of quinolones (in combination with ceftazidime: $\mathrm{SBPI}_{\mathrm{CAZ}+\mathrm{MXF}}=2.125, \mathrm{SBPI}_{\mathrm{CAZ}+\mathrm{LEV}}=4.125$ ) and colistin (in combination with quinolons: $\mathrm{SBPI}_{\mathrm{COL}+\mathrm{MXF}}=2.125$ ) decreased into the susceptible range, and only in one isolate out of the tested four ones. 


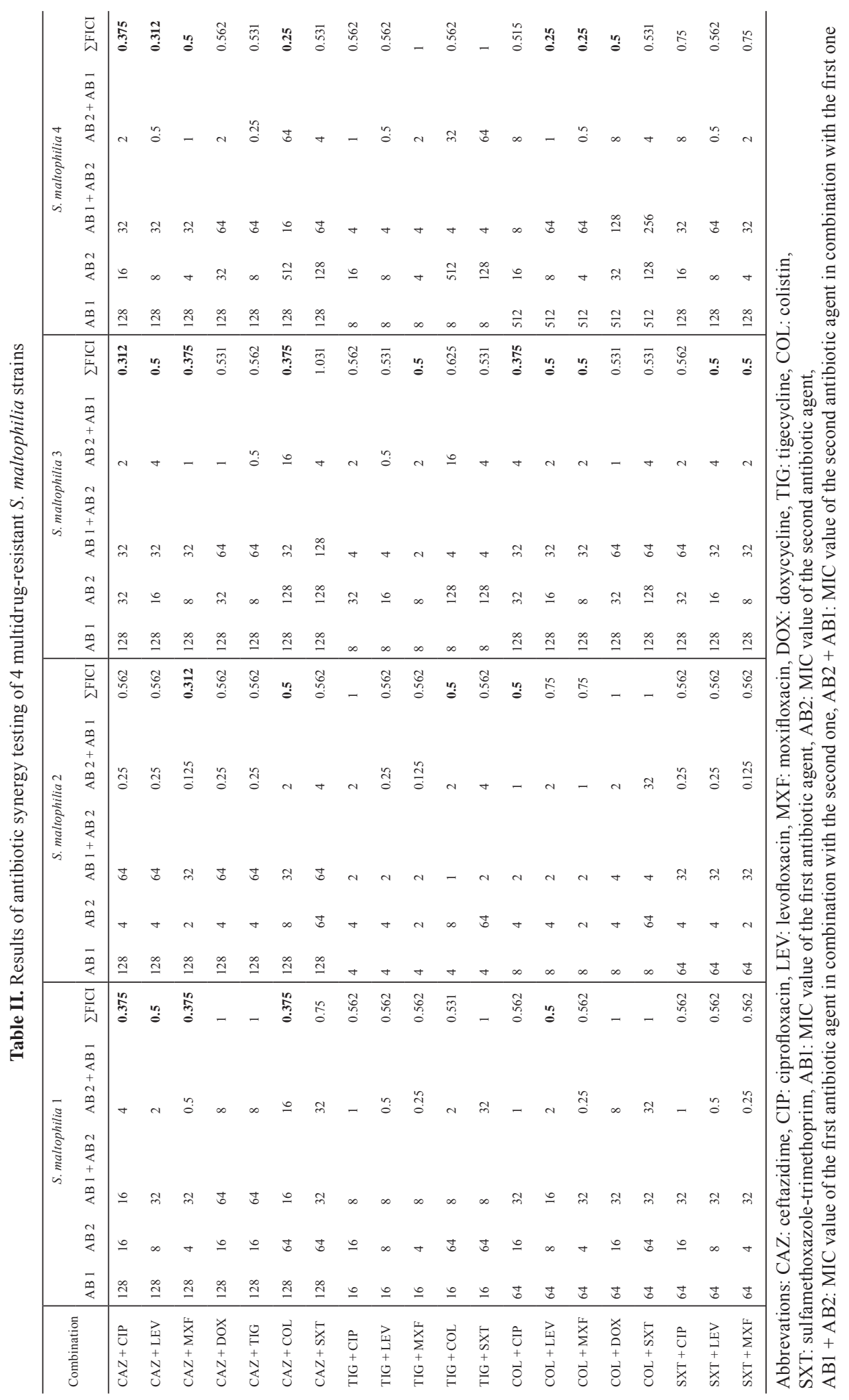


Genetic testing was performed only in 27 strains out of the 30. Except of two isolates carrying sul2, all the others were positive for sull. None of the strains contained both genes. Our study found that 7 strains (23\%) carried a class 1 integron when integrase-specific primers were used. ERIC-PCR resulted in diverse patterns, but 4 identical patterns among the isolates ( 2 times 2 isolates, once 3 isolates and once 9 isolates with the same band pattern) were found. Despite the detected clonality, isolates with the same band pattern showed different MIC values. All isolates were included in the final evaluation of antibiotic susceptibility testing results.

\section{Discussion}

The frequency of SXT-resistant $S$. maltophilia isolates was found to be $2 \%$ in our centre [18]. Despite this low frequency and the dominantly colonizing presence, emergence of resistance to SXT in S. maltophilia means a real challenge. Comparing the detected MIC values to our previous results, frequency of antibiotic resistance was found to be higher in SXT-resistant S. maltophilia strains than in SXT-susceptible ones [18]. Even if the resistance rate was 20-23\% to levofloxacin and moxifloxacin, these newer fluoroquinolones mean real therapeutic options. Both can disrupt $S$. maltophilia biofilms $[1,2]$. It was demonstrated that moxifloxacin possesses considerable time-kill effect on SXT-resistant S. maltophilia [19]. The high achievable concentration of quinolones in respiratory tract by applying them as inhalation therapy makes these antibiotic agents more valuable. As it was revealed from the final clinical reports retrospectively, 4 out of the 7 infected patients were successfully treated with levofloxacin or moxifloxacin. Susceptibility for doxycycline was investigated instead of the often tested minocyclin, since the latter has not been distributed in Hungary. Doxycycline looked to be the most effective antibiotic agent in this study, but it has to be emphasized that this result was based on S. maltophilia specific ECOFF. It is known that ECOFF and well-established clinical breakpoints can differ significantly; so it has to be assessed if this agent is a real therapeutic option. However, our result is in concordance with a previous study, in which doxycycline was found to be the most active agent against SXT-resistant $S$. maltophilia strains isolated from CF patients [10]. For the interpretation of tigecycline, the lowest breakpoints were applied. With other breakpoints (Enterobacteriaceae specific ones) the interpreted results of tigecycline susceptibility testing would have been more favourable. Even if chloramphenicol is a recommended drug according to CLSI and $13 \%$ of SXT-resistant S. maltophilia strains were susceptible in this study, the severe adverse effects of this drug must be born in mind. 
Combination therapy is recommended for severe invasive infections, for immunocompromised patients and for empirical therapy in areas with high frequency of local resistance against SXT [4]. Combinations can be more active than monotherapy and can reduce the risk of developing antibiotic resistance during treatment, but superiority of combination therapy is not proved [1]. Clinical trails are missing yet. Stenotrophomonas maltophilia infections are often polymicrobial, where the use of a combination therapy may be also advantageous [14]. Against biofilm-growth $S$. maltophilia isolates combinations were in vitro effective, too [20]. In case of extremely drug-resistant $S$. maltophilia infections, combination therapy can be useful (maybe the only) therapeutic alternative. Several studies tested in vitro antibiotic combinations on SXT-susceptible S. maltophilia isolates, but only few ones focused on SXT-resistant ones [21]. By different methods, a lot of synergistic combinations were described; including those we tested $[8,11,22-25]$. According to our results, synergy is strain dependent. Ceftazidime and colistin can be synergistic with quinolones. The level of colistin or quinolones in aerosolised form can achieve 4-5 times higher concentration in airways than in serum during respiratory tract infections. Therefore, an antibiotic agent in aerosolised form in combination with an intravenous one can be effective, even if the FIC and SBPI do not show synergism [26].

Limitation of our study is that results gained by checkerboard method were confirmed only by combined E-test method (BioMérieux) [27]. Multiplecombination bactericidal testing and time-kill assay were not performed. However, there is no true gold standard for synergy testing. The literature suggests that no two synergy methods produce comparable results. Moreover, the true clinical relevance of synergy testing is unknown because of missing outcomebased studies [27]. A further limitation is the number of combinations tested. Many other combinations, including ticarcillin-clavulanate, aztreonam, azithromycin, rifampin, etc. could have been tested. We focused just on the most commonly used and available antibiotic agents in Hungary.

Methodically, synergic combinations detected by checkerboard method could be detected also by combined E-test method in our study. Maybe the E-test method is more practical in the daily routine in a busy diagnostic laboratory, but it requires very precise work. Moreover, the filmy growth of S. maltophilia microcolonies can make the evaluation of this test difficult. For antibiotic susceptibility testing of S. maltophilia, disc diffusion cannot be applied (except SXT), but synergic effects might be detected by double disc diffusion assay [14]. Ceftazidime plus levofloxacin or moxifloxacin synergy was detected also by this method in our study, but only tight inhibition zones were seen, not the typical keyhole shape. 
Twenty-five isolates carried sul1, but only 7 were positive for class 1 integron with intI primers. It is possible that class I integrons may not have the same 5' and 3' ends, as described by Hu et al. [7]. Therefore the absence of a class 1 integron could not be confirmed. In combination with $d f r$ A and sul2 genes, sul 1 gene can lead to high resistance to SXT as well as multidrug resistance. While sull genes were associated with class 1 integrons and sul2 genes were found on plasmids, the mobilisation of sul genes is probable and likely to increase with SXT consumption. Ongoing resistance surveillance is needed [28].

In summary, SXT-resistant $S$. maltophilia strains are rarely isolated in our laboratory and the most of the isolates are colonizers. We concluded that levofloxacin and moxifloxacin are the most effective antibiotic agents against multidrug-resistant $S$. maltophilia. Combinations including fluoroquinolones especially moxifloxacin - can be effective, even if the isolate was resistant to them. It has to be kept in mind that results of synergy testing are isolate specific.

\section{Authors' Contributions}

$\mathrm{JE}$ and $\mathrm{KK}$ conceived and designed the experiments. JE performed the experiments, analysed the data, wrote and corrected the manuscript. PJ and IM contributed in collecting the isolates and in correction of the manuscript. KK contributed reagents/materials.

\section{Conflict of Interest}

The authors declare that they have no competing interests.

\section{References}

1. Abbott, I. J., Slavin, M. A., Turnidge, J. D., Thursky, K. A., Worth, L. J.: Stenotrophomonas maltophilia: Emerging disease patterns and challenges for treatment. Expert Rev Anti Infect Ther 9(4), 471-488 (2011).

2. Brooke, J. S.: Stenotrophomonas maltophilia: An emerging global opportunistic pathogen. Clin Microbiol Rev 25(1), 2-41 (2012).

3. http://www.eucast.org/clinical_breakpoints/

4. Looney, W. J., Narita, M., Mühlemann, K.: Stenotrophomonas maltophilia: An emerging opportunist human pathogen. Lancet Infect Dis 9(5), 312-323 (2009). 
5. Barbolla, R., Catalano, M., Orman, B. E., Famiglietti, A., Vay, C., Smayevsky, J., Centrón, D., Piñeiro, S. A.: Class 1 integrons increase trimethoprim-sulfamethoxazole MICs against epidemiologically unrelated Stenotrophomonas maltophilia isolates. Antimicrob Agents Chemother 48(2), 666-669 (2004).

6. Chang, L. L., Lin, H. H., Chang, C. Y., Lu, P. L.: Increased incidence of class 1 integrons in trimethoprim/sulfamethoxazole-resistant clinical isolates of Stenotrophomonas maltophilia. J Antimicrob Chemother 59(5), 1038-1039 (2007).

7. Hu, L. F., Chang, X., Ye, Y., Wang, Z. X., Shao, Y. B., Shi, W., Li, X., Li, J. B.: Stenotrophomonas maltophilia resistance to trimethoprim/sulfamethoxazole mediated by acquisition of sul and dfrA genes in a plasmid-mediated class 1 integron. Int $\mathrm{J}$ Antimicrob Agents 37(3), 230-234 (2011).

8. Hu, L. F., Gao, L. P., Ye, Y., Chen, X., Zhou, X. T., Yang, H. F., Liiu, Y. Y., Mei, Q., Li, J. B.: Susceptibility of Stenotrophomonas maltophilia clinical strains in China to antimicrobial combinations. J Chemother 26(5), 282-286 (2014).

9. Brooke, J. S.: New strategies against Stenotrophomonas maltophilia: A serious worldwide intrinsically drug-resistant opportunistic pathogen. Expert Rev Anti Infect Ther 12(1), 1-4 (2014).

10. San Gabriel, P., Zhou, J., Tabibi, S., Chen, Y., Trauzzi, M., Saiman, L.: Antimicrobial susceptibility and synergy studies of Stenotrophomonas maltophilia isolates from patients with cystic fibrosis. Antimicrob Agents Chemother 48(1), 168-171 (2004).

11. Milne, K. E., Gould, I. M.: Combination antimicrobial susceptibility testing of multidrug-resistant Stenotrophomonas maltophilia from cystic fibrosis patients. Antimicrob Agents Chemother 56(8), 4071-4077 (2012).

12. Clinical and Laboratory Standards Institute. Performance Standards for Antimicrobial Susceptibility testing. CLSI approved Standard. M100 S16. CLSI, Wayne, PA, USA, 2012.

13. Balke, B., Hogardt, M., Schmoldt, S., Hoy, L., Weissbrodt, H., Häussler, S.: Evaluation of the E test for the assessment of synergy of antibiotic combinations against multiresistant Pseudomonas aeruginosa isolates from cystic fibrosis patients. Eur J Clin Microbiol Infect Dis 25(1), 25-30 (2006).

14. Betts, J. W., Phee, L. M., Woodford, N., Wareham, D. W.: Activity of colistin in combination with tigecycline or rifampicin against multidrug-resistant Stenotrophomonas maltophilia. Eur J Clin Microbiol Infect Dis 33(9), 1565-1572 (2014).

15. Garcia, L. S.: Clinical Microbiology Procedures Handbook. 3rd Edition, ASM Press, Washington DC, 2010, Chapter 5.12.

16. Silbert, S., Pfaller, M. A., Hollis, R. J., Barth, A. L., Sader, H. S.: Evaluation of three molecular typing techniques for nonfermentative Gram-negative bacilli. Infect Control Hosp Epidemiol 25(10), 847-851 (2004).

17. Grape, M., Farra, A., Kronvall, G., Sundström, L.: Integrons and gene cassettes in clinical isolates of co-trimoxazole-resistant Gram-negative bacteria. Clin Microbiol Infect 11(3), 185-192 (2005).

18. Juhász, E., Krizsán, G., Lengyel, G., Grósz, G., Pongrácz, J., Kristóf, K.: Infection and colonization by Stenotrophomonas maltophilia: Antimicrobial susceptibility and clinical background of strains isolated at a tertiary care centre in Hungary. Ann Clin Microbiol Antimicrob 13(1), 333 (2014).

19. Giamarellos-Bourboulis, E. J., Karnesis, L., Galani, I., Giamarellou, H.: In vitro killing effect of moxifloxacin on clinical isolates of Stenotrophomonas maltophilia resistant to trimethoprim-sulfamethoxazole. Antimicrob Agents Chemother 46(12), 3997-3999 (2002). 
20. Wu, K., Yau, Y. C., Matukas, L., Waters, V.: Biofilm compared to conventional antimicrobial susceptibility of Stenotrophomonas maltophilia isolates from cystic fibrosis patients. Antimicrob Agents Chemother 57(3), 1546-1548 (2013).

21. Giamarellos-Bourboulis, E. J., Karnesis, L., Giamarellou, H.: Synergy of colistin with rifampin and trimethoprim/sulfamethoxazole on multidrug-resistant Stenotrophomonas maltophilia. Diagn Microbiol Infect Dis 44(3), 259-263 (2002).

22. Church, D., Lloyd, T., Peirano, G., Pitout, J.: Antimicrobial susceptibility and combination testing of invasive Stenotrophomonas maltophilia isolates. Scand J Infect Dis 45(4), 265-270 (2013).

23. Visalli, M. A., Jacobs, M. R., Appelbaum, P. C.: Activities of three quinolones, alone and in combination with extended-spectrum cephalosporins or gentamicin, against Stenotrophomonas maltophilia. Antimicrob Agents Chemother 42(8), 2002-2005 (1998).

24. Entenza, J. M., Moreillon, P.: Tigecycline in combination with other antimicrobials: A review of in vitro, animal and case report studies. Int J Antimicrob Agents 34(1), 8.e1-9 (2009).

25. Gülmez, D., Cakar, A., Sener, B., Karakaya, J., Hasçelik, G.: Comparison of different antimicrobial susceptibility testing methods for Stenotrophomonas maltophilia and results of synergy testing. J Infect Chemother 16(5), 322-328 (2010).

26. Wood, G. C., Underwood, E. L., Croce, M. A., Swanson, J. M., Fabian, T. C.: Treatment of recurrent Stenotrophomonas maltophilia ventilator-associated pneumonia with doxycycline and aerosolized colistin. Ann Pharmacother 44(10), 1665-1668 (2010).

27. Doern, C. D.: When does 2 plus 2 equal 5? A review of antimicrobial synergy testing. J Clin Microbiol 52(12), 4124-4128 (2014).

28. Toleman, M. A., Bennett, P. M., Bennett, D. M., Jones, R. N., Walsh, T. R.: Global emergence of trimethoprim/sulfamethoxazole resistance in Stenotrophomonas maltophilia mediated by acquisition of sul genes. Emerg Infect Dis 13(4), 559-565 (2007). 